\title{
Advanced Visualization for OLAP
}

\author{
Andreas S. Maniatis ${ }^{1}$ \\ Tel. +30-210-7721436 \\ andreas@dblab.ntua.gr
${ }^{1}$ National Technical University of Athens
Dept. of Elec. and Computer Eng.
9, Iroon Polytechneiou St.
15780 Athens, Hellas

\author{
Spiros Skiadopoulos ${ }^{1}$ \\ Tel. +30-210-7721402 \\ spiros@dblab.ntua.gr
}

Yannis Vassiliou $^{1}$
Tel. +30-210-7722526
yv@cs.ntua.gr

${ }^{2}$ University of loannina
Dept. of Computer Science
45110 loannina, Hellas

\begin{abstract}
Data visualization is one of the big issues of database research. OLAP as a decision support technology is highly related to the developments of data visualization area. In this paper we demonstrate how the Cube Presentation Model (CPM), a novel presentational model for OLAP screens, can be naturally mapped on the Table Lens, which is an advanced visualization technique from the Human-Computer Interaction area, particularly tailored for cross-tab reports. We consider how the user interacts with an OLAP screen and based on the particularities of Table Lens, we propose an automated proactive users support. Finally, we discuss the necessity and the applicability of advanced visualization techniques in the presence of recent technological developments.
\end{abstract}

\section{Categories and Subject Descriptors}

H.2.1 [Database Management]: Logical Design - data models. H.2.3 [Database Management]: Languages - report writers H.5.2 [Information Interfaces and Presentation]: User Interfaces - graphical user interfaces (GUI), user-centered design.

General Terms: Design, Human Factors.

Keywords: On-Line Analytical Processing, Visualization

\section{INTRODUCTION}

In the last years, On-Line Analytical Processing (OLAP) and data warehousing have become major research areas in the database community [3,7]. Although the modeling of data [16,17] has been extensively dealt with, an equally important issue of the OLAP domain, the visualization of data, has not been adequately investigated. In the context of OLAP, data visualization deals with the techniques and tools used for presenting OLAP specific information to end-users and decision makers. The database community expects visualization to be of significant importance in the area, during the next years [7], and although research has provided results dealing with the presentation of vast amounts of

\footnotetext{
Permission to make digital or hard copies of all or part of this work for personal or classroom use is granted without fee provided that copies are not made or distributed for profit or commercial advantage and that copies bear this notice and the full citation on the first page. To copy otherwise, or republish, to post on servers or to redistribute to lists, requires prior specific permission and/or a fee.

DOLAP '03, November 7, 2003, New Orleans, Louisiana, USA.

Copyright 2003 ACM 1-58113-727-3/03/0011 ..\$5.00.
}

data $[5,4,1,15]$, OLAP has not been part of advanced visualization techniques so far.

In this paper, we start by adopting a newly introduced presentation model for OLAP called Cube Presentation Model - CPM [11] and demonstrate how it can be combined with non-traditional visualization techniques. The CPM model distinguishes representation from data retrieval. It is separated in two layers: a logical that deals with data retrieval and representation and a presentational that provides a generic model for data representation. In this paper, we present a quick informal overview of the main characteristics of CPM and accompany them with its respective UML modeling for ease of understanding. Then, we proceed with the contributions of this paper, which can be listed as follows:

- Initially, we present a mapping of the generic presentational scheme of CPM to the particularities of an advanced visualization technique coming from the field of Human Computer Interaction. The Table Lens technique [14,12] is particularly tailored for cross-tab reports, which are most commonly used for OLAP purposes and it is accompanied by a set of handy features for the exploration of data sets which are presented in this way.

- Next, we provide algorithms for the automated proactive support of the user during his interaction with an OLAP screen, based on the particularities of Table Lens. Specifically, Table Lens employs a particular distortion of the presentation to highlight areas of increasing interest to the user. We provide a generic algorithm to support this task proactively.

- Finally, we discuss the necessity and the applicability of such visualization techniques in the presence of current technological developments.

The remainder of this paper is structured as follows. In Section 2, we summarize the logical and the presentation layers of CPM. Section 3 shows how CPM can be naturally combined with Table Lens. Moreover, Section 3 demonstrates the automate proactive support to the user. In Section 4, we discuss the necessity and applicability of the proposed ideas. Finally, Section 5 concludes our results and presents topics for future work. A longer version of this paper, with more details can be found in [8].

\section{THE CUBE PRESENTATION MODEL}

Although OLAP has been an active research area for the past few years, the efforts devoted to the visualization of OLAP screens are very scarce. To our knowledge, only two such efforts exist [10,1]. The first is from the industrial field, where Microsoft has issued a 
commercial standard for multidimensional databases and where the presentational issues form a major part [10]. In this approach, a powerful query language is used to provide the user with complex reports, created from several cubes (or actually subsets of existing cubes). The second is an academic approach, the Tape Model [1], based on the notion of "Tapes", called so due to their look and feel. Tapes are infinite and can overlap (if they contain shared data dimensions), or intersect with each other. A two dimensional intersection is called a matrix and represents a kind of cross-tab between the corresponding dimensions. Each tape comprises of a variable number of tracks. The most important operations on tapes include: (a) insertion and deletion of tracks, (b) changing the sequence of tracks (i.e., sorting) and (c) scrolling on tracks. The Tape Model offers the possibility of defining hierarchical structures within a tape.

In [11], we have presented the Cube Presentation Model (CPM), a novel proposal towards a presentation model for OLAP screens. CPM is composed of two parts: (a) a logical layer which involves the formulation of cubes and (b) a presentational layer that involves the presentation of these cubes (normally, on a 2D screen). The main idea behind CPM lies in the separation of logical data retrieval (which we encapsulate in the logical layer of CPM) and data presentation (captured from the presentational layer of CPM). This duality provides the flexibility of possibly replacing one of the two layers with an alternative proposal smoothly. The logical layer that we propose is based on an extension of a previous proposal [18] with additional functionality that allows us to incorporate more complex cubes. In a nutshell, the logical model involves (a) dimensions defined as lattices of dimension levels, (b) ancestor functions (in the form of $\mathrm{anc}^{\mathrm{L}_{1} ; \mathrm{L}_{2}}$ ) mapping values between related levels of a dimension, (c) detailed data sets, practically modeling fact tables at the lowest granule of information for all their dimensions and (d) cubes, defined as aggregations over detailed data sets. In this paper, we will not deal with the formal presentation of the underlying logical layer of CPM (the reader is referenced to [11] for a detailed and in depth presentation) but focus on the mapping of our presentation layer to alternative visualization techniques from the area of Human Computer Interaction.

Following, we give an intuitive and informal description of the presentation layer of CPM that provides a formal model for OLAP screens.

The most important entities - as far as display aspects are concerned - of the presentation layer of CPM include:

- Points: A point over an axis resembles the classical notion of points over axes in mathematics. In the simple case, a point is characterized by an equality selection condition over a level (e.g., City=Seattle). Nevertheless, as we shall see, we can multiplex several logical dimensions to one presentational axis; therefore, a point will be formally defined to handle this kind of situations, too.

- Axis: An axis can be viewed as a set of points. We introduce two special purpose kinds of axes, Invisible and Content. An Invisible axis is a placeholder for the levels of the data set which are to be presented to the user. The Content axis has a more elaborate role: it is a place holder for the content of the multicube, as computed over the detailed data.

- Multicubes. A multicube is defined over (a) a multidimensional space, comprising a set of axes, (b) an underlying data set providing all the data which will be filtered and aggregated before presented to the user and (c) a mapping among the multidimensional space and the underlying data set that shows the computation of the multicube contents.

- 2D-slice: A 2D slice is a 2D layer of data that can be presented on the screen. Consider a multicube $\mathrm{MC}$, composed of $\mathrm{K}$ axes. A $2 D$-slice over $M C$ can be sufficiently defined by a set of $(\mathrm{K}-2)$ points, each from a separate axis. Intuitively, a $2 \mathrm{D}$-slice pins the axes of the multicube to specific points, except for 2 axes, which will be presented on the screen (or a printout). In Fig. 2, we depict such a 2D slice over a multicube. //check whether it is the MS picture...

- Tape: Intuitively, a tape is column or a row over a 2D-slice, i.e., a construct parallel to an axis. Again, if we consider a 2D-slice SL over a multicube MC, composed of $K$ axes, a tape is sufficiently defined by a set of $(\mathrm{K}-1)$ points, where the $(\mathrm{K}-2)$ points are the points of SL. A tape is always parallel to a specific axis: out of the two "free" axis of the 2D-slice, we pin one of them to a specific point which distinguishes the tape from the 2D-slice.

- Cross-join: Intuitively, if we take one tape parallel to the horizontal axis and another parallel to the vertical axis, their intersection is a cell. In the most general case, as we shall see, it can be a set of cells. In both cases, the intersection of two nonparallel tapes is called a cross-join. Consider a 2D-slice SL over a multicube $\mathrm{MC}$, composed of $K$ axes and two tapes $t_{1}$ and $t_{2}$ which are not parallel to the same axis. A cross-join over $t_{1}$ and $t_{2}$ is defined by a set of $K$ points, where the $(K-2)$ points are the points of SL and each of the two remaining points is a point on a different axis of the remaining axes of the slice.

- Content Function: At the schema level, we assume a function assigning the computation of measures to the Content axis of the multicube, along with ordering and other restrictions. We also assume a function, mapping combinations of multicube coordinates, one from each of the coordinate axis of the multicube to the measure axis. Each such assignment is practically a row in the result set of one of the queries/expressions/... computing the multicube, which we call cell ${ }^{1}$. For brevity, in the sequel, we simply tag the Content axis with this information.

To make the discussion easier, we will use an example taken from [10], throughout the paper (Figure 1). In this example, we assume a cube Salescube is defined over the dimensions Products, Salesman, Time, and Geography, each involving several levels of aggregation. In this query, we restrict the Time dimension to the sales of Year 1991. We ignore the Products dimension (Product $\mathrm{S}=\mathrm{ALL}$ ) in the subsequent aggregation of detailed data. Whenever we need to present a $2 \mathrm{D}$ screen and more than two dimensions are involved, we need to merge (CROSSJOIN in [10] terminology) as many dimensions as necessary in a single axis. In this case, we combine the dimensions Salesman (restricted on two

\footnotetext{
1 The name cell stems from the regular terminology of OLAP, referring to points in the multidimensional space. Although in the classical tabular representation of data, cell is actually a successful name, for other representation techniques this does not apply (e.g., in the proposal of [4], a cell should be represented by a line).
} 
salesmen) and Geography on the COLUMNS axis and leave the dimension Time on the ROWS axis. Note that the Geography dimension involves more than one levels of aggregation (both City and Region). The same applies for the Time dimension, where both Quarters and Months are employed.

In terms of CPM terminology, the query of Figure 1 is a 2D-Slice, say SL (see also Figure 2). In SL one can identify 4 horizontal tapes denoted as R1, R2, R3 and R4 in Figure 1) and 6 vertical tapes (numbered from $\mathrm{C} 1$ to $\mathrm{C} 6$ ). The meaning of the horizontal tapes is straightforward: they represent the Quarter dimension, expressed either as quarters or as months. The meaning of the vertical tapes is somewhat more complex: they represent the combination of the dimensions Salesman and Geography, with the latter expressed in City, Region and Country level. Moreover, two constraints are superimposed over these tapes: the Year dimension is pinned to a specific value (i.e., Year=1991) and the Product dimension is ignored. One can also consider the cross-join $t 1$ defined by the common cells of the tapes R1 and C1.

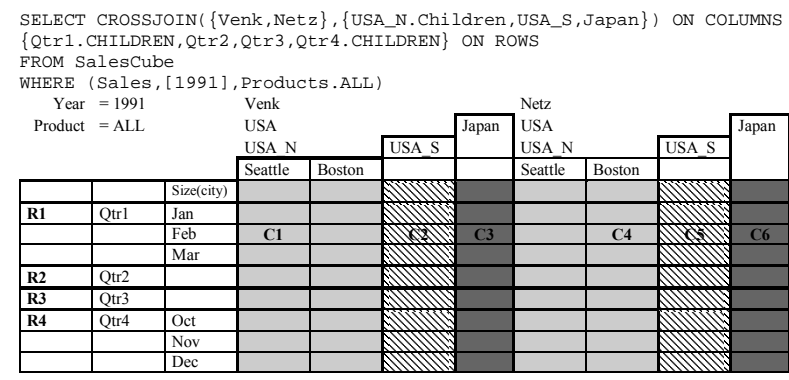

Figure 1. Motivating example for the cube model [10,11].

Interpreting our motivating example in terms of CPM, we assume a detailed data set, named Salescube, under the schema:

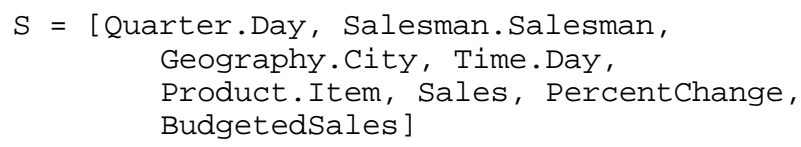

The following axis schemata can also be discerned in Figure 2:

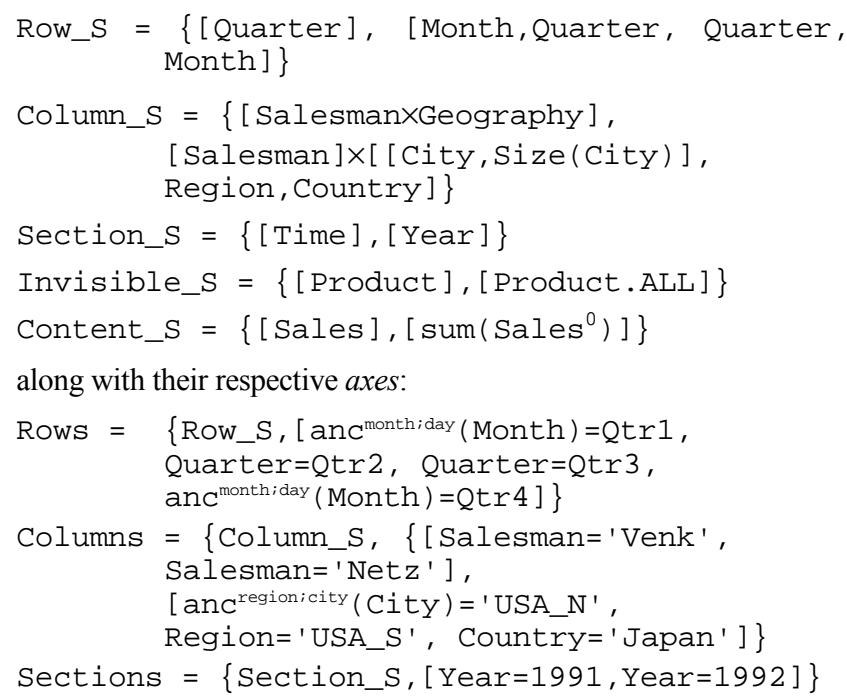

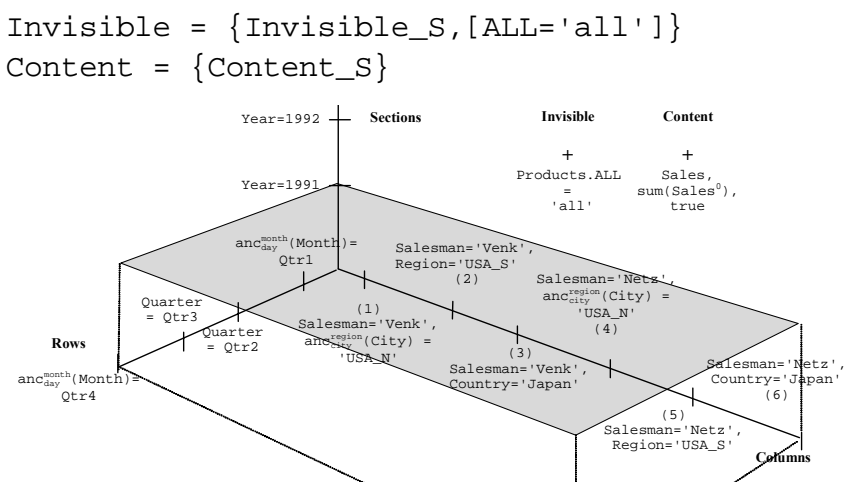

Figure 2. The 2D-Slice SL for the example of Figure 2 [11].

In Figure 2, we can also observe an exemplary point over an axis, incorporating equality conditions for each of the involved dimensions of the axis:

$$
\begin{gathered}
\mathrm{p}_{1}=([\text { Salesman, }[\underline{\text { City }}, \operatorname{Size}(\text { City) }]], \\
{\left[\text { Salesman='Venk', } a n C^{\text {region; city }}(\text { City })=\text { 'USA_N'] }^{\prime}\right)}
\end{gathered}
$$

Thus, a multicube MC can be defined as:

$$
\mathrm{MC}=\{\text { Rows, Columns, Sections, Invisible, }
$$

Finally, in Figure 3, we present some more comprehensive visualization representations of multicubes, axes, points, 2D slices and cross-joins on a 3D and 2D layout.

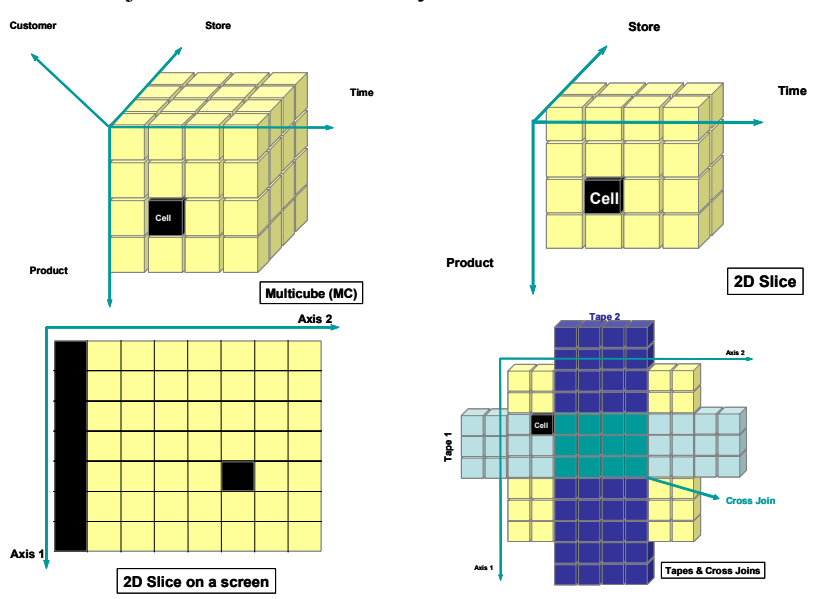

Figure 3. Mapping CPM objects to 3D and 2D Cross Tabular layouts.

\section{MAPPING CPM TO VISUALIZATION TECHNIQUES}

In this section, we will demonstrate how CPM can be combined with Table Lens (TL) [14,12], a traditional cross-tabular presentation model from the Human Computer Interaction area. This model is widely used in applications and platforms for the visualization of tabular, multivariate and multidimensional data and appears to be quite appropriate for OLAP purposes. Table Lens is based on the "focus plus context" technique that allows visualizing 
and manipulating large 2-D tables [14]. Using Table Lens, we can easily examine patterns and correlations in large tables and effectively zoom in without losing the global picture of our data. We have chosen Table Lens as an advanced visualization technique due to the fact that it is based on a cross-tabular paradigm for the presentation of information; a paradigm quite popular in OLAP screens, too.

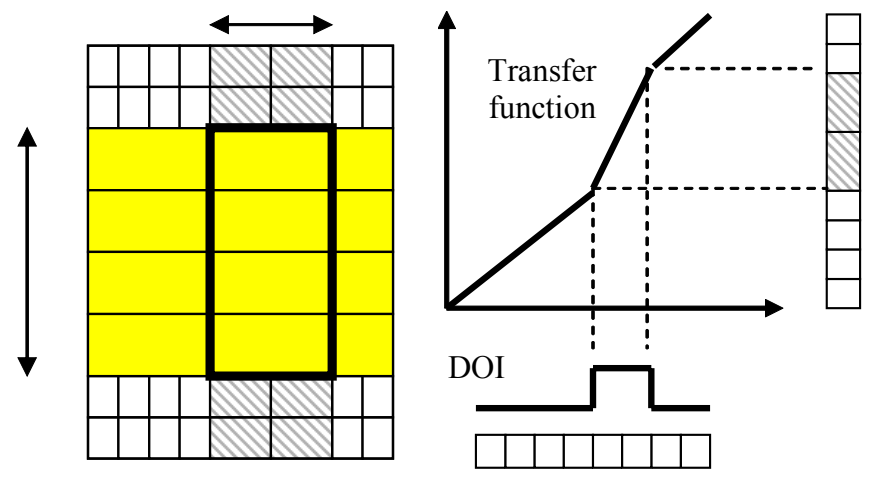

Figure 4. A Table Lens example: (a) a $2 \times 4$ focus window is defined over a space of $8 \times 8$ points; (b) Table Lens distortion of the Columns axis

\subsection{Mapping CPM to Table Lens}

In this subsection, we will present the main features of Table Lens, and then we will link it to the CPM model. The main constructs of the Table Lens technique involve:

- Axes: The Table Lens model assumes two axes. For clarity, we will use Rows and Columns to denote these two axes.

- 2D space: The 2D space is constructed from the Cartesian product of the two Table Lens axes. It is a (finite) matrix of cells.

- Degree of Interest Function (DOI): DOI is a function that maps each axis point to a value that indicates the level of interest for that point. For each axis a different DOI function is prescribed.

- Transfer Function: A transfer function maps each cell to its physical locations, indicating the level of zoom for each cell. Practically, the transfer function is the translation of the respective DOI function (operating at the "interest" space) to the "pixel" space.

One of the basic ideas behind the Table Lens technique is that not all cells are considered equal. In fact, certain cells comprising a concrete region of the 2D space are assigned to occupy more surface of the screen than the rest of the cells. This is the essence of zooming into the particular region of the $2 \mathrm{D}$ space. To implement this, in the simplest setting of Table Lens, each DOI function is a simple "pulse" function, meaning that it has a standard value for all points, except for the points of a certain interval that are mapped to a higher value. Remember that each axis has its own DOI function, thus a $2 \mathrm{D}$ space is characterized by $2 \mathrm{D}$ windows of focus. In Figure $4 \mathrm{a}$, we depict an $8 \times 8$ space with a $2 \times 4$ focus window. In Figure $4 \mathrm{~b}$ we show (i) how the originally equally important cells of the Columns axis are assigned importance values by the DOI function: notice the pulse on two particular cells that assigns them greater importance than the rest of the cells and (ii) how the Transfer function, defined as a weighted integral of the DOI function maps the points to pixel areas. For reasons of efficient representation [14], in Figure $4 \mathrm{~b}$, the produced axis is rotated by $90^{\circ}$. Finally, another interesting feature of Table Lens is the ability to define more than one windows of focus. This is quite helpful in situations where two areas can be contrasted and compared. As we shall see in the next section, this feature is particularly useful in the case of OLAP.

There is an easy way to map the underlying constructs of the CPM to the ones of the Table Lens. The axis points of CPM are mapped to axis points of Table Lens and a 2D slice in CPM is implemented as a $2 \mathrm{D}$ space in Table Lens. The contents function provides the values of the cells of the 2D space. Naturally, CPM is generic enough to lack the particularities of the axis distortion due to the DOI function. The naïve way to overcome the limitation is simply to ask the user to define a certain window of focus over the presented 2D slice, specifying both its size and position. Still, we can automate the process on the basis of the structure and the contents of a $2 \mathrm{D}$ slice.

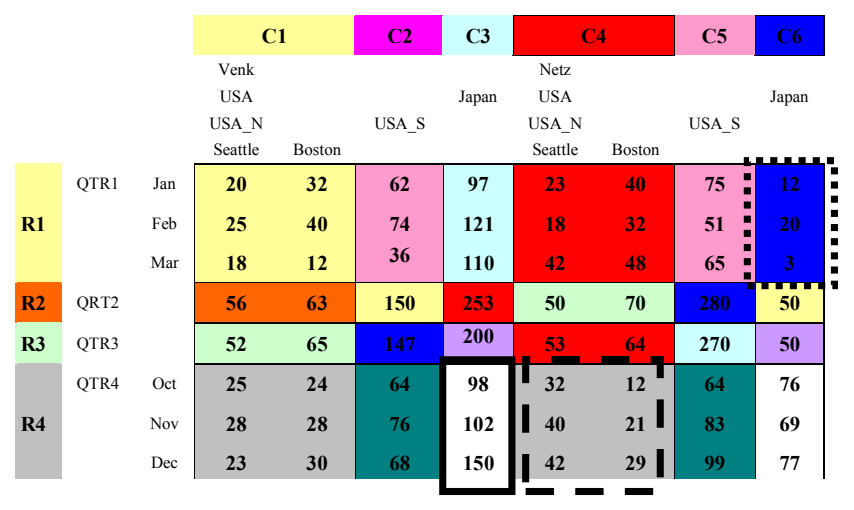

Figure 5. Instantiation of the motivating example with values; different shading determine different cross-joins and thick borders highlight the cross-joins with the highest, lowest and closest to average values.

\subsection{Which Window of Interest to Choose?}

In this subsection, we will deal with the problem of providing the user with proactive automated support for the exploration of an OLAP report. Our main tool towards this end is the window of interest as determined by the DOI functions and the basic idea is to provide an algorithm to proactively determine the window of interest over a $2 \mathrm{D}$ slice. We want to define an algorithm that automatically determines this window whenever a user invokes an OLAP report. It appears that we can come up with a generic algorithm, where the stopping conditions, error range and other parameters can be tuned by the user. Actually, we can even treat as a parameter a choice on whether the user is simply interested of having a window of a certain surface or he is actually interested to see a focus on a range of cells satisfying certain statistical properties (e.g., minimum/maximum/closest to average set of values). Having determined algorithmically the window of interest, the two involved DOI functions, which are independent from each other, are directly derived.

\subsection{Motivation and Assumptions}

Before providing the generic algorithm, let us clarify our contribution through a specific example. We instantiate the example of Figure 2 with the values of Figure 5. Let us assume that when the 
user activates this OLAP screen, he would like to be informed on three particular cross-joins: one involving the maximum sales, another involving the lowest and a third involving the cross-join with behavior closest to the average of the whole screen. Practically, this involves three windows of focus, which we depict through a thick border around the involved cross-joins. In this particular case, the cross-join R1/C6 is the one with the lowest summary of values, the cross-join $\mathrm{R} 4 / \mathrm{C} 4$ the one with the highest sum and the cross-join $\mathrm{R} 2 / \mathrm{C} 3$ is the one closest to the average sales per cross-join (which amounts to 240.5 sales per cross-join).

A vanilla algorithm to compute the aforementioned quantities proceeds as follows: (a) summarizes all cells per cross-join; (b) sorts cross-joins and computes the average cross-join value and (c) pinpoints the three regions of interest. This algorithm has linear (precisely, one-pass) complexity on the number of cells and $n \log n$ (due to sorting) complexity on the number of cross-joins. Actually, if we are simply to keep the max, min or closest-to-avg cross-join, a linear single pass from all the cells is sufficient, without any sorting. In the case of avg, each time that we summarize the cells from a cross-join we can compute the average of the individual cross-join summaries and compute the closest cross-join to the current value of this average.

Assumptions: Underlying this proactive notification to the user, we have made the following assumptions:

- Cross-joins constitute homogeneous pieces of information. This means that we can assume a certain level of semantic cohesion among the cells of a certain cross-join. Moreover, we can assume that each cross-join can be considered as a distinct semantic unit and that cross-joins are comparable to each other. For example, we assume that it makes sense to compare sales from Japan to the sales of Southern USA. Naturally, the user choices for the axes points (and the produced cross-joins) may severely affect this assumption.

- Statistically speaking, we are allowed to perform certain aggregate operations over our data. Specifically, we assume that the underlying detailed data set has been summarized by a distributive aggregate function.

In [6] aggregation functions are categorized as (a) distributive functions, like max, min, sum or count, meaning that there is a way to compute the result of the application of the aggregation function to the overall data set by composing the individual results of its application to subsets of the dataset; (b) algebraic functions that are expressed as finite algebraic expressions over distributive functions, like avg; and (c) holistic functions for all other functions.

To forestall any possible criticism, we want to point out that the exact result of aggregation operations over a 2D slice is handled by the logical layer. In the case of the [18] model, all operations are formally defined as operations over the detailed data set; optimization results for the obvious cases are also provided. Nevertheless, in the case of this paper, we want a quick approximation of the statistical measures under consideration, to be used for the determination of the focus window and not of the values of the report. Thus, problems like the Simpson's paradox or the non invariance property [6] are considered as out of the scope of this paper. Finally, as a general comment, since it is quite cumbersome to ask the user each time to characterize the statistical nature of his underlying data, we employ the idea that one can have an indication of the statistical nature of the information of screen by observing the aggregate function that has been applied to compute them. Thus, since in our case we are starting with a sum aggregate function, we conclude that we can apply further distributive operations to the measure Sales in order to obtain our indicative approximations.

\subsection{A Generic Algorithm for Determining the Window of Focus}

Naturally, we can do better than the aforementioned vanilla algorithm by adding extra criteria to the proactive selection of the starting window of focus. We propose a guided greedy generic algorithm, GenericFocusWindow (Figure 6), to deal with the issue. The simple idea underlying the algorithm is that there are certain conditions to be met for the focus window. For example, one could require that the focus window occupies at most/least a certain percentage of the screen size, or of a certain size of cells. Moreover, the selected window optimizes an objective function. The property Determining Quality of the algorithms captures exactly this requirement in the form of a certain function. Since our algorithm is greedy, we need an Original Pick routine to start the processing; in general this is closely related to the Determining Quality function and we require that it starts with a smallest value. Moreover, a Guard Condition checks for the satisfaction of the desired property (meaning that we can possibly allow a certain approximation error $\varepsilon$ to out obtained solution). Finally, a function Pick provides the necessary details for working from the original small-in-value solution towards the final result, practically picking the next crossjoin to enlarge the current window of focus.

One implicit assumption that our algorithm makes is that the Original Pick fits inside the allowed window. This constraint can easily be relaxed by an extension of the algorithm picking subparts of a cross-join in a similar fashion with the proposed algorithm, if we consider that we pick subparts of a 2Dslice. For lack of space we do not incorporate this extension too.

We will give two examples for the instantiation of the aforementioned generic algorithm. In the first case (Algorithm FocusWindow Min $3 \times 3$ in Figure 7), we are interested in a focus window which (a) includes the window with the minimum summary of values and (b) is not bigger than $3 \times 3$ (with a tolerance of the surface $\varepsilon=1$ ). We can observe that the guided greedy algorithm picks the window of minimum value as its starting point. The first constraint is met by the original pick and the second by the stop condition of the algorithm. During the expansion phase, each time we choose a cross-join such that (a) it is neighbouring with the current solution; (b) if merged with the current solution, it comprises a rectangle (easily determined by comparing the lengths of the opposite sides of the new solution and (c) has the smallest surface.

If we execute the algorithm on the data of Figure 5, the result will be $\mathbf{Q}=\{\mathrm{R} 1 / \mathrm{C} 6, \mathrm{R} 2 / \mathrm{C} 6, \mathrm{R} 3 / \mathrm{C} 6, \mathrm{R} 4 / \mathrm{C} 6\}$ which is practically the tape $\mathrm{C} 6$. If instead of the minimum value, in function Pick we had chosen the maximum, then the result would be $\mathbf{Q}=\{\mathrm{R} 1 / \mathrm{C} 6, \mathrm{R} 1 / \mathrm{C} 5\}$. Another obvious extension would be to employ a 2-greedy algorithm: in this case the small cross-joins R2 / C5 and R2 / C6, each comprising a single cell, could have been incorporated in the solution too. 


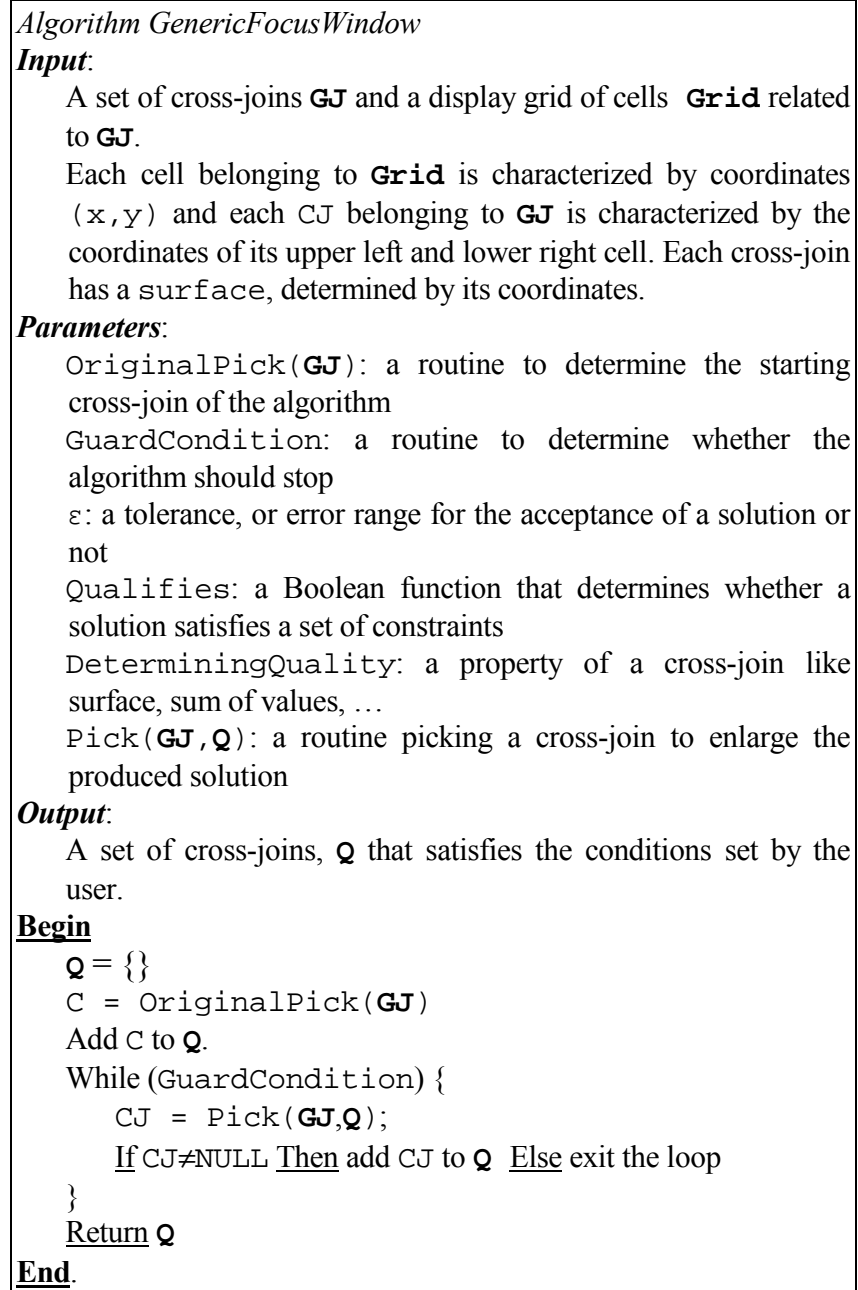

A set of cross-joins GJ and a display grid of cells Grid related to GJ.

Each cell belonging to Grid is characterized by coordinates $(\mathrm{x}, \mathrm{y})$ and each $\mathrm{CJ}$ belonging to $\mathbf{G J}$ is characterized by the coordinates of its upper left and lower right cell. Each cross-join has a surface, determined by its coordinates.

\section{Parameters:}

OriginalPick (GJ): a routine to determine the starting cross-join of the algorithm

GuardCondition: a routine to determine whether the algorithm should stop

$\varepsilon$ : a tolerance, or error range for the acceptance of a solution or not

Qualifies: a Boolean function that determines whether a solution satisfies a set of constraints

DeterminingQuality: a property of a cross-join like surface, sum of values, ...

Pick $(\mathbf{G J}, \mathbf{Q})$ : a routine picking a cross-join to enlarge the produced solution

Output:

A set of cross-joins, $\mathbf{Q}$ that satisfies the conditions set by the user.

Begin

$$
\mathbf{Q}=\{\}
$$

$\mathrm{C}=$ OriginalPick (GJ)

Add $\mathrm{C}$ to $\mathbf{Q}$.

While (GuardCondition) \{

$\mathrm{CJ}=\operatorname{PiCk}(\mathbf{G J}, \mathbf{Q})$;

If $\mathrm{CJ} \neq \mathrm{NULL}$ Then add $\mathrm{CJ}$ to $\mathrm{Q}$ Else exit the loop

\}

$\underline{\text { Return } \mathrm{Q}}$

End.

Figure 6. Algorithm GenericFocusWindow

In a different example (Figure 8), we also demonstrate an algorithm producing a focus window which (a) includes the window with the maximum summary of values and (b) is not bigger than $3 \times 3$ (with a tolerance of the surface $\bullet=0$ ) and (c) optimizes a combined formula over surface and value. Each time we pick the cross-joins that brings us closest to the $3 \times 3$ desideratum, while having the highest summary value. In the case of our motivating example, the solution is $\mathbf{Q}=\{\mathrm{R} 4 / \mathrm{C} 3, \mathrm{R} 4 / \mathrm{C} 4\}$ with an exact $3 \times 3$ surface.

\section{DISCUSSION}

At this point, we would like to take the time to discuss the larger framework of the contribution of this paper. First, as the Lowell report [7] mentions, visualization is one of the big issues of database research for the next years. To copy from the Lowell report, "The original Laguna-Beach report lamented that there was little research on user interfaces to DBMSs. ... There have not been comparable advances in the last 15 years. There is a crying need for better ideas in this area". We claim that of all fields of database research, decision support and OLAP are the ones to be affected most out of this phenomenon.
Algorithm FocusWindow_Min_3x3

Input:

A set of cross-joins GJ and a display grid of cells Grid related to GJ.

Each cell belonging to Grid is characterized by coordinates $(x, y)$ and each $\mathrm{CJ}$ belonging to $\mathrm{GJ}$ is characterized by the coordinates of its upper left and lower right cell. Each cross-join has a surface, determined by its coordinates.

\section{Parameters:}

OriginalPick(GJ): start with cross-join having the minimum summary

GuardCondition $(\mathbf{Q}, \varepsilon)$ : the surface is closest to $3 \times 3$

$\varepsilon: 1$ cell $^{2}$

Qualifies: a Boolean function that determines whether a solution satisfies a set of constraints

DeterminingQuality $(\mathbf{2})$ : distance from the ideal $3 \times 3$ surface

Pick $(\mathbf{G J}, \mathbf{Q})$ : a routine picking the cross-join with minimum distance from the ideal $3 \times 3$ surface

Output:

A set of cross-joins, $\mathbf{Q}$ that satisfies the conditions set by the user.

Begin

$\mathbf{Q}=\{\}$

$\mathbf{C}=$ OriginalPick (GJ)

Add $C$ to $Q$.

While (GuardCondition) \{

$\mathrm{CJ}=\mathrm{Pick}(\mathrm{GJ}, \mathbf{Q})$;

If $\mathrm{CJ} \neq \mathrm{NULL}$ Then add $\mathrm{CJ}$ to $\mathrm{Q}$ Else exit the loop \}

Return $Q$

End.

OriginalPick (GJ)

Let the cross-join $C_{r}$ s.t., $\left|\operatorname{sum}\left(C_{r}\right)\right|$ is the minimum;

Among equals pick the upper and left-wise;

$\underline{\text { Return }}\left(\mathrm{C}_{\mathrm{r}}\right)$;

\}

DeterminingQuality (Q)

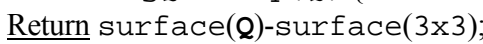

\}

GuardCondition $(\mathbf{Q}, 1)\{$

If surface( $(\mathbf{Q})$-surface $(3 \times 3)<1$ Then Return true;

Else Return false

\}

Pick $(\mathbf{C J}, \mathbf{Q})\{$

Let $\mathbf{v}$ be the subset of the cross-joins of $\mathbf{C J}$, s.t., for each $v \in \mathbf{v}$ : Qualifies $(\mathbf{v}, \mathbf{C J}, \mathbf{Q})$

Let $\mathrm{V}_{\mathrm{P}} \in \mathbf{V}$ be a cross-join s.t., DeterminingQuality (Q) is minimum, if $\mathrm{V}_{\mathrm{P}}$ is added to $\mathbf{Q}$.

$\underline{\text { Return }} \mathrm{v}_{\mathrm{P}}$

\}

Qualifies $(\mathrm{V}, \mathbf{C J}, \mathbf{Q})\{$

If ( $\mathrm{v}$ is adjacent to a cross-join $\mathrm{CJ} \in \mathbf{C} \boldsymbol{J}) \quad \& \&$

( $v \cup \mathrm{Q}$ forms a rectangle)

Then Return true;

Else Return false

Figure 7. Algorithm FocusWindow_Min_3x3 


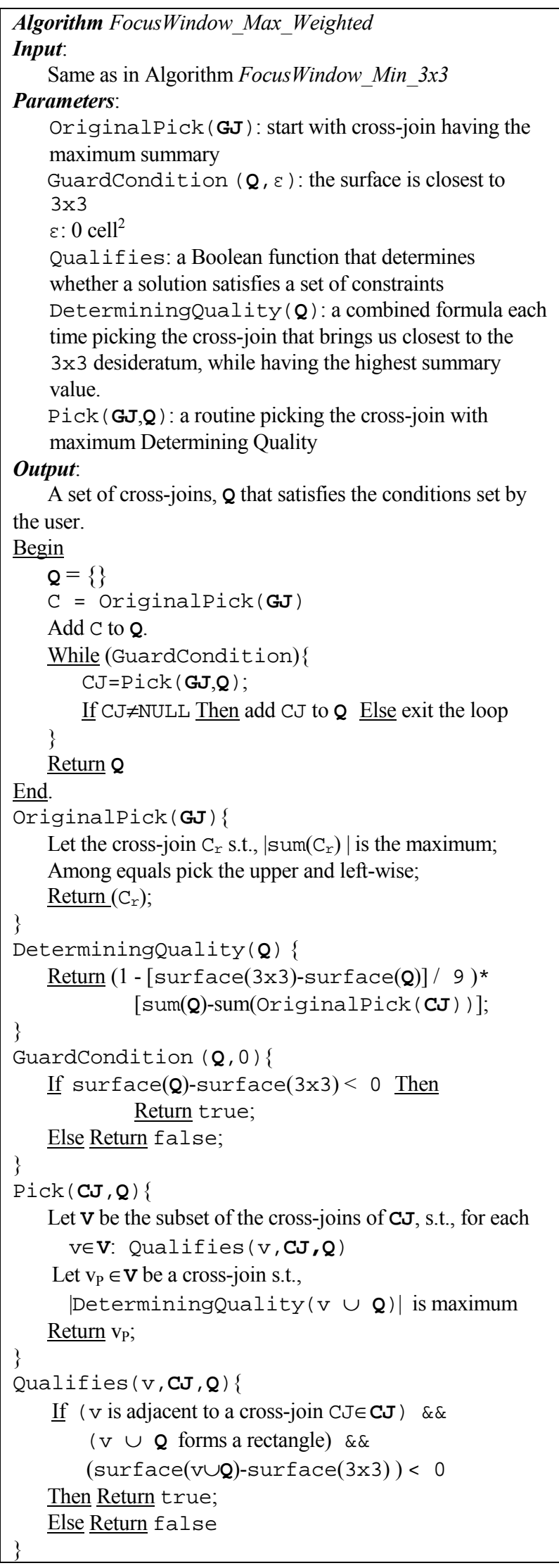

Figure 8. Algorithm FocusWindow_Max_Weighted
Someone could possibly question the need for a new model. For us it is clear that one of the main reasons for the research community not dealing with visualization issues so far, is the heritage of the computing paradigm of the past three decades. This paradigm silently made the assumption that the user sitting in front of a console makes one query and retrieves one answer (as would have happened in a UNIX terminal thirty years ago). This is not the case with modern user interfaces for datasets, especially in the context of OLAP. The user makes simultaneously many queries, combined in one or more screens; nevertheless, all our modeling techniques and languages so far (from the relational model, to SQL and the OLAP modeling efforts proposed in the academia) simply ignore this fact. Our effort tries to formalize the simultaneous presence of more than one queries and this is done in two layers. In the presentational layer we provide a uniform and generic model for the user interface, which hides the complexity of answer retrieval, detached in the logical layer. As a second interesting difference, note that the users work in sessions of queries, as opposed to sequences of unrelated queries. OLAP is a typical, but not the only, case for this behavior.

As a first attempt towards the issue, we have carefully selected a visualization technique from the fields of Human-Computer Interaction and Information Visualization with the particularity of being crafted specifically for tabular data and we have customized it for OLAP. Naturally, we do not claim that this is the ultimate solution to the problem, but rather we wish to indicate that there is quite an interesting research field in this area and a supportive body of knowledge from other disciplines, such as Human-Computer Interaction and Information Visualization.

At the same time, new hardware developments pose new requirements for our visualization techniques. One of our goals is to implement OLAP visualization techniques for particularly small devices such mobile phones and palmtops. Although the processing power of these gadgets is no more negligible (actually, the buzzword 'thin client' seems to disappear from the standard vocabulary of the area) their screen sizes shrink over time. To make OLAP screens presentable to such devices one can follow several paths, such as: (a) show only high level summaries which involve small 2D slices or (b) show simply pie- or bar-charts. We choose an alternative approach where (a) the contents of the screen do not have to be squeezed in size in order to fit in the screen, and most importantly (b) the report does not have to be rewritten neither do we have to check for the aggregation level of the presented data. On the contrary, a certain part of the report is presented depending on the particularities of the device. Here, we make the reasonable assumption that either the device has the computational power to determine the amount of cells that can be presented to the user or, if this is not an option, the device can at least piggy-back its characteristics to the OLAP server and let the server decide on the focus window.

Third, making the discussion a little broader, we bring up the Table Lens technique to highlight the possibility of making proactive user decision support in the presence of large datasets (in our case, the value axis is quite larger than the size that someone can handle efficiently). Clearly, as report screens are limited not only due to hardware constraints, but also due to the particularities of human nature (e.g., the classical discussion on the limited capacity of persons in processing information [9]), it comes quite natural that automated proactive support to the users is thus one of the new requirements that decision support tools have to provide. Thus, this 
end of our contribution is related to a broader line of research $[2,13]$.

\section{CONCLUSIONS AND FUTURE WORK}

In this paper, we have demonstrated how the Cube Presentation Model, a novel presentation model for OLAP data can be naturally mapped into an advanced visualization technique, the Table Lens. Initially, we have defined the mapping scheme from the Cube Presentation Model to Table Lens entities and objects. Then, we have introduced suitable algorithms for proactive automated support of the user towards the highlighting of interesting areas of a report. Finally, we have discussed on the usefulness and applicability of the proposed techniques to modern technological developments.

Next steps in our research include the introduction of suitable, CPM specific, visualization techniques that comply to current standards and recommendations as far as usability and user interface design is concerned and its extension to address the specific visualization requirements of mobile and wireless OLAP, as this notion can be supported and implemented on mobile devices and palmtops.

\section{REFERENCES}

[1] M. Gebhardt, M. Jarke, S. Jacobs: A Toolkit for Negotiation Support Interfaces to Multi-Dimensional Data. ACM SIGMOD 1997, pp. $348-356$.

[2] J. Han. Towards On-Line Analytical Mining in Large Databases. SIGMOD Record, 27(1): 97-107, 1998.

[3] W.H. Inmon: Building the Data Warehouse. John Wiley \& Sons, 1996.

[4] Alfred Inselberg: Visualization and Knowledge Discovery for High Dimensional Data. $2^{\text {nd }}$ Workshop Proceedings UIDIS, IEEE, 2001.

[5] D.A. Keim. Visual Data Mining. Tutorials of the $23^{\text {rd }}$ International Conference on Very Large Data Bases, Athens, Greece, 1997.

[6] Hans-J. Lenz, Bernhard Thalheim. OLAP Databases and Aggregation Functions. In Proc. of the $13^{\text {th }}$ International Conference on Scientific and Statistical Database Management (SSDBM'01), 2001.
[7] Various Authors: The Lowell Database Research Self Assessment. Lowell, Massachusetts USA, May 4-6, 2003. Available at: http://research.microsoft.com/ Gray/lowell/.

[8] Andreas Maniatis, Panos Vassiliadis, Spiros Skiadopoulos, Yannis Vassiliou: Advanced Visualization for OLAP (long version).

[9] George A. Miller. The magical number seven, plus or minus two: Some limits on our capacity for processing information. Psychological Review, 63, 81-97 (1956).

[10] Microsoft Corp. OLEDB for OLAP February 1998. Available at: http://www.microsoft.com/data/oledb/olap/.

[11] Andreas Maniatis, Panos Vassiliadis, Spiros Skiadopoulos, Yannis Vassiliou: CPM: A Cube Presentation Model for OLAP. DaWaK 2003, Prague, Czech Republic, September 3 52003.

[12] Peter Pirollo, Ramana Rao: Table Lens as a Tool for Making Sense of Data. Proceedings of the AVI '96 Workshop, Gubbio, Italy, June 1996.

[13] S. Sarawagi, R. Agrawal, N. Megiddo: Discovery-Driven Exploration of OLAP data Cubes, Proceedings of the $6^{\text {th }}$ International Conference on Extending Database Technology (EDBT'98), Valencia, Spain, March 1998.

[14] Ramana Rao, Stuart K. Card: The Table Lens: Merging Graphical and Symbolic Representations in an effective Focus + Context Visualization for Tabular Information. Proceedings of the ACM SIGCHI (CHI '94), Boston, Massachusetts USA, April 24-28, 1994.

[15] Thomas Ruf, Juergen Georlich, Ingo Reinfells: Dealing with Complex Reports in OLAP Applications. DaWaK '99, Florence, Italy, August $30^{\text {th }}-$ September $1^{\text {st }} 1999$.

[16] Aris Tsois, Nikos Karayannidis, Timos Sellis: $M A C$ : Conceptual Data Modeling for OLAP. Proc. of the International Workshop on DMDW 2001.

[17] P. Vassiliadis, T. Sellis: A Survey on Logical Models for OLAP Databases. SIGMOD Record, vol. 28, no. 4, December 1999.

[18] Panos Vassiliadis, Spiros Skiadopoulos: Modeling and Optimization Issues for Multidimensional Databases. Proc. of CAiSE'00, Stockholm, Sweden, 2000. 